
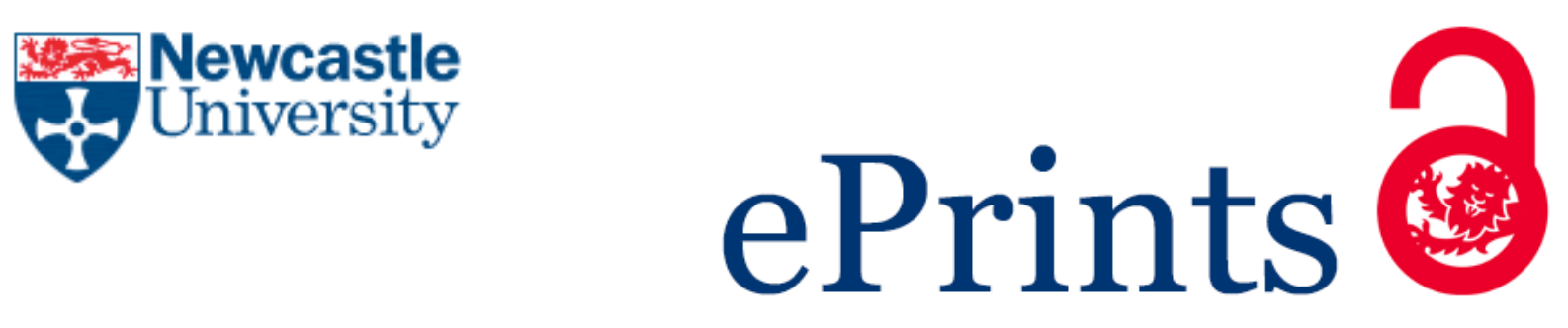

Godfrey A, Bourke A, Del Din S, Morris R, Hickey A, Helbostad J, Rochester L. Towards holistic free-living assessment in Parkinson's disease: unification of gait and fall algorithms with a single accelerometer.

In: 38th Annual International Conference of the IEEE Engineering in Medicine and Biology Society (EMBC).

16-20 August 2016, Orlando, Florida: IEEE.

(C) 2016 IEEE. Personal use of this material is permitted. Permission from IEEE must be obtained for all other uses, in any current or future media, including reprinting/republishing this material for advertising or promotional purposes, creating new collective works, for resale or redistribution to servers or lists, or reuse of any copyrighted component of this work in other works.

Conference website:

http://embc.embs.org/2016/

Date deposited:

$22 / 06 / 2016$ 


\title{
Towards holistic free-living assessment in Parkinson's disease: unification of gait and fall algorithms with a single accelerometer
}

\author{
Alan Godfrey, Member, IEEE, Alan Bourke, Silvia Del Din, Member, IEEE, Rosie Morris, Aodhán \\ Hickey, Jorunn L Helbostad, and Lynn Rochester
}

\begin{abstract}
Technological developments have seen the miniaturization of sensors, small enough to be embedded in wearable devices facilitating unobtrusive and longitudinal monitoring in free-living environments. Concurrently, the advances in algorithms have been adhoc and fragmented. To advance the mainstream use of wearable technology and improved functionality of algorithms all methodologies must be unified and robustly tested within controlled and free-living conditions. Here we present and unify a (i) gait segmentation and analysis algorithm and (ii) a fall detection algorithm. We tested the unified algorithms on a cohort of young healthy adults within a laboratory. We then deployed the algorithms on longitudinal (7 day) accelerometer-based data from an older adult with Parkinson's disease (PD) to quantify real world gait and falls. We compared instrumented falls to a self-reported falls diary to test algorithm efficiency and discuss the use of unified algorithms to impact free-living assessment in PD where accurate recognition of gait may reduce the number of automated detected falls (38/week). This informs ongoing work to use gait and related outcomes as pragmatic clinical markers.
\end{abstract}

Presented at the $38^{\text {th }}$ Annual International Conference of the IEEE Engineering in Medicine and Biology Society, Orlando, Florida, USA

Conference: http://embc.embs.org/2016/

16-20 August 2016

Available on IEEEXplore.

\section{Introduction}

Wearable technology through the use of inertial sensors has the ability to positively impact the care and treatment of users in modern healthcare [1]. However, the pragmatic deployment of these devices remains ad-hoc and limited to bespoke research projects [2]. This is primarily attributed to the lack of robust analytic signal/data interpretation (algorithms) from free-living data sets. Moreover, algorithms have been developed in isolation, on particular physiological functions or events relating to a serious/adverse event, i.e. spatio-temporal gait events, postural transitions, balance, falls [3-5]. Yet, it has been shown that a single accelerometer-based device worn on the lower back can be utilized to capture a range of supervised physical capability tasks while implementing a number of algorithms [6]. However, of notable importance are gait and fall detection algorithms where pragmatic free-living deployment remains limited as it is difficult to (i) identify/segment all gait events (and to date the literature remains vague on how to achieve/define this) and (ii) accurately detect falls from challenging environments due to habitual behaviours in agreement with a self-reported fall(s). The combination of these algorithms seems logical due to their clinical importance and functional dependency: identification of upright events (possible gait) to determine the gravitational vector (to identify a fall).

Therefore, the purpose of this pilot study was to harmonise gait and fall algorithms toward a more holistic assessment of free-living PD from longitudinal accelerometer data (7 day). This forms part of ongoing work aiming to quantify free-living gait as a surrogate marker of cognitive decline in idiopathic Parkinson's disease (PD) involving a comprehensive evaluation of gait, balance, cognitive function, and falls.

\section{A. Gait segmentation}

\section{Related work}

Free-living gait segmentation is challenging due to the heterogeneity of ambulatory activity within habitual environments. To date, studies have examined purposeful bouts/periods of gait $[7,8]$ by applying thresholds ( 10 and 60 s), yet this excludes up to $97 \%$ of all gait bouts [9]. We present a novel segmentation algorithm with the added provision of macro behavioural outcomes (e.g. time walking) and micro outcomes (e.g. step time) during the process.

Supported by Newcastle University Institute for Ageing (NUIA) Responsive Mode and the Engineering and Physical Sciences Research Council (EPSRC), number EP/N508718/1. AG and LR are supported by the National Institute for Health Research (NIHR) Newcastle Biomedical Research Centre (BRC) and Unit (BRU) based at Newcastle upon Tyne Hospitals NHS Foundation Trust and Newcastle University. Work also supported by the NIHR Newcastle Clinical Research Faculty infrastructure funding and LiveWell a collaborative grant from the Lifelong Health and Wellbeing initiative, managed by the Medical Research Council (MRC), number G0900686. The views expressed are solely those of the authors.

A. Godfrey, S. Del Din, R. Morris, A. Hickey and L. Rochester are with the Institute of Neuroscience, Newcastle University, Newcastle upon Tyne, NE4 5PL, UK (phone: +44-191-208-1245; fax: +44-191-208-1250; e-mail: alan.godfrey@ncl.ac.uk, silvia.del-din@ncl.ac.uk, rosie.morris@ncl.ac.uk, aodhan.hickey@ncl.ac.uk, lynn.rochester@ncl.ac.uk).

A. Bourke and J Helbostad are with the Department of Neuroscience, Norwegian University of Science and Technology, Trondheim, Norway (alan.bourke@ntnu.no, jorunn.helbostad@ntnu.no). 


\section{B. Macro and micro gait}

Utilising the continuous nature of an accelerometer signal, free-living gait has been defined according to a macro and micro conceptual model [10]. This can be achieved in two stages by (i) examining the broad trends in the signal to quantify the behavioural (macro) characteristics of gait including total walking time and distributions of bouts [11] and (ii) identifying the initial/final contact (IC/FC) events of the gait cycle within bouts from the filtered peaks/troughs of the raw signal $[3,12]$ to quantify spatio-temporal outcomes sensitive to age/pathology including step time, step length, step time variability/asymmetry [13].

\section{Fall detection}

Falls within PD are poorly understood with no validated quantitative outcomes in free-living and are among the most incapacitating features of PD, negatively impacting quality of life. Algorithms have been developed based on magnitude thresholds of accelerations differentiating between normal activities of daily living (ADL) and fall impact [5, 14]. More astute algorithms utilising impact, velocity and posture have achieved $100 \%$ specificity and sensitivity with a false-positive of $<1$ per day [15]. While the latter utilised data on older adults (>65yrs) over extended periods (8hrs) these were non fallers or those with PD. Therefore, deployment on those cohorts is paramount to test algorithm performance and utility during longitudinal assessment.

\section{Fall diaries and classification}

Currently, falls diaries are the accepted 'gold standard' for falls quantification. Yet this traditional method of self-report underestimates total number of falls due to perceived definition of a fall or fear or reporting due to associated stigma. This can lead to incorrect patient treatment and increased demands on family/healthcare professionals. Classification systems attribute falls to participant-centric or environmental factors but recent interest has focused on the importance of the context of falls, in particular the physical action undertaken by the faller immediately prior to falling [16], i.e. 'pre-fall event' [17]. Objective identification of a fall and assessment of a pre-fall event with accelerometer-based methodologies remains untested.

\section{Methods}

\section{A. Gait segmentation algorithm}

The segmentation algorithm initially relies on logical heuristics and a moving window to define bouts of upright movement based on the standard deviation (SD) and mean of the vertical acceleration with the use of predefined thresholds. Based on device location (lower back) this identified bouts of: (i) upright and not moving (sitting/standing still) and (ii) upright and moving (possible walking). Once the start/end of an upright and moving bout was identified the segmented data were investigated for possible gait events: corrected for misalignment [18] and subjected to a validated continuous wavelet transform (CWT) technique to identify IC/FC within the gait cycle [19]. Bouts with no IC/FC events were discarded. A new bout of walking was defined if there was no step detected after $2 \mathrm{~s}$ (greater than 1 stride time). The correct identification of a walking bout directly informed the fall algorithm, which relied upon a known upright bout to estimate the gravitational vector.

\section{B. Macro and micro gait algorithm}

Once the segmentation process identifies gait bouts, macro (bout number, bout length, distribution) gait outcomes can be estimated. The segmenting process also quantifies 14 micro spatio-temporal characteristics, achieved through the sequence of IC/FC events in relation to the double support phase of the gait cycle, previously validated within PD [19].

\section{Fall algorithm}

The optimum configuration for fall detection utilises: impact, identified from the root-sum-of-squares (RSS) of the tri-axial accelerations; vertical velocity estimate, numerical integration of the RSS signal with the magnitude of static acceleration (gravity) subtracted; and posture, determined by taking the dot product of the reference gravity vector and the current gravity vector estimate relative to the body segment [15]. The posture of lying is detected if the angle exceeds $60^{\circ}$ for more than $75 \%$ of the duration.

\section{Fall diaries and classification}

Falls diaries were examined from the Incidence of Cognitive Impairment in Cohorts with Longitudinal Evaluation-GAIT (ICICLE-GAIT) study, aiming to better understand the mechanisms determining the transition from PD to PD with dementia in an incident cohort. ICICLE-GAIT routinely assesses participants at 18 month intervals, including 7 day free-living with accelerometry. A selection of diaries was examined for overlap between self-reported fall(s), while wearing an accelerometer.

\section{Evaluation}

Algorithms were implemented during scripted activities (inc. gait and falls) with healthy adults within a laboratory setting and later used on 7 day free-living PD data.

\section{A. Stage I}

Ten healthy adults $\left(33.7 \pm 4.7\right.$ years, $\left.22.2 \pm 3.0 \mathrm{~kg} / \mathrm{m}^{2}, 5 \mathrm{M} / 5 \mathrm{~F}\right)$ were recruited. Ethical consent was granted by the National Research Ethics Service (County Durham and Tees Valley) and the Newcastle-upon-Tyne Hospitals NHS Foundation Trust (11/NE/0383). All participants gave informed written consent before starting.

Each participant wore a tri-axial accelerometer-based device (AX3, Axivity, York, UK: $23.0 \times 32.5 \times 7.6 \mathrm{~mm}$, weight $9 \mathrm{~g}$, $100 \mathrm{~Hz}, 16$-bit, $\pm 8 \mathrm{~g}$ ) located on the fifth lumbar vertebrae (L5). The device was held in place by double sided tape and Hypafix (BSN Medical Limited, Hull, UK). Recorded signals were stored locally on internal memory (512MB, raw binary file), later 
downloaded upon completion of each trial. Additionally, video recordings (Sony HandyCam DCR-SR77) were captured during each trial.

Participants performed purposeful walks along with a number of scripted tasks in the following order: sitting, standing, repeated sit-to-stand-to-sit $(\times 3)$, repeated stand-to-sit-lying (supine, left, right) to-sit-to-stand $(\times 3), 12 \mathrm{~m}$ intermittent walk $(\times 4)$, 2 minute walking $(\times 3$, self-selected: preferred, fast and slow), walking up/down steps $(\times 3)$ and falls from a standing posture onto $40 \mathrm{~mm}$ thick mats. Participants were instructed to fall forward (Fig. 1A), backward (1B), right (1C) and left (1D) with knees flexion. Each fall direction was performed 3 times. Participants were instructed to use their arms to protect themselves during impact. It was noted when short 'non-purposeful' walks (inc. steps in a fixed location) were performed between each of those tasks as the participant moved to different locations within the laboratory or self-preparation between tasks.

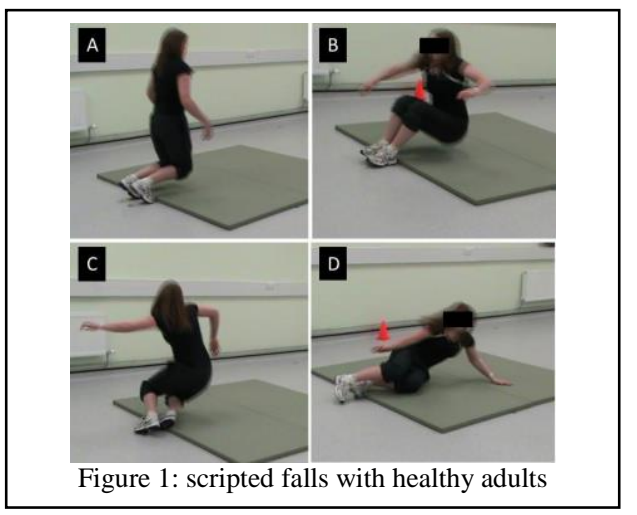

\section{B. Stage II}

Ethical approval for ICICLE-GAIT was granted by the Newcastle and North Tyneside research ethics committee. All participants gave informed consent form prior to testing. Falls diaries within the ICICLE-GAIT study were examined for selfreported fall(s). Cross over between self-reported falls was rare but one participant was found and included for the purposes of this pilot work (M, 73.4 years, $27.8 \mathrm{~kg} / \mathrm{m}^{2}$ ). Clinical outcomes were extracted from the PD participant, including: self-efficacy freezing of gait (FOG), balance confidence using the self-rated Activities Balance Self Confidence Scale (ABC), severity of PD motor symptoms using the Hoehn \& Yahr scale (H\&Y: 0 no symptoms to 5 wheelchair bound or bedridden), and section III of the modified Movement Disorder Society version of the Unified Parkinson's Disease Rating Scale (MDS-UPDRS, 0 no motor symptoms to 132 severe motor symptoms). The participant wore the same device as described previously, with the same attachment methods. The device was worn continuously for 7 days (24h/day), only removed during bathing. Replacement adhesives and tape to re-attach were provided.

\section{Results}

\section{A. Stage I- Gait segmentation}

The gait segmentation algorithm correctly identified (100\%) all purposeful bouts (tasks) of gait, including stair ascent and descent, from other scripted tasks and random noise in the signal prior to use (i.e. handling and non-wear on L5) for all participants, Fig. 2A, 2B and 3.

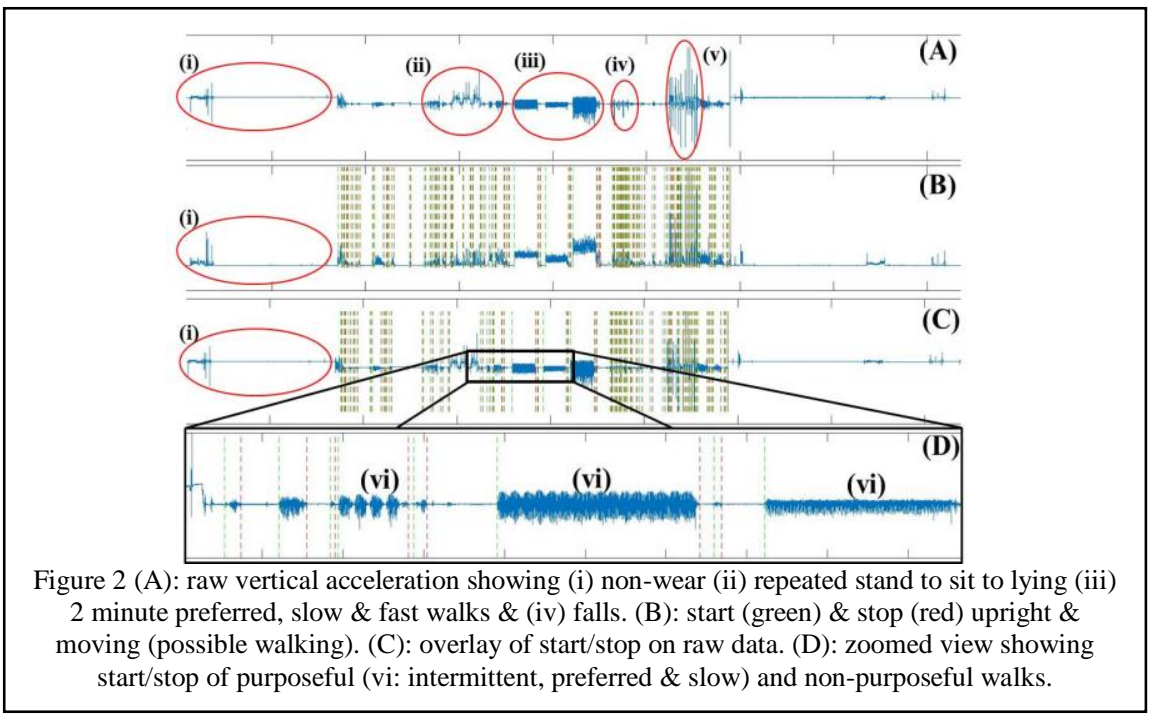

The promising feature of this segmentation method is the ability to identify and quantify the short (termed 'non-purposeful' for this pilot work) bouts of gait, Figure 2D. Quantification of 'non-purposeful' bouts were consistent with examination of the 
raw acceleration signals as well as general undertaking of the complete protocol, i.e. sequence of events between purposeful tasks, Fig. 2 and 3.

However, during the falls it was observed that while the segmentation algorithm was able to segment gait before/after the participant completed the fall task, in some instances the fall event was within the segmented bout, Fig. 3.

\section{B. Stage I-Macro and micro}

Though the gait segmentation algorithm defined periods of purposeful/non-purposeful gait (macro: bout number and length) its implementation through IC/FC detection also enables the quantification of step count and micro gait characteristics (e.g. step time, stance time variability, swing time asymmetry), previously validated within the 2 minute purposeful bout of preferred walking $[19,20]$. Macro and micro outcomes will be presented in other work.

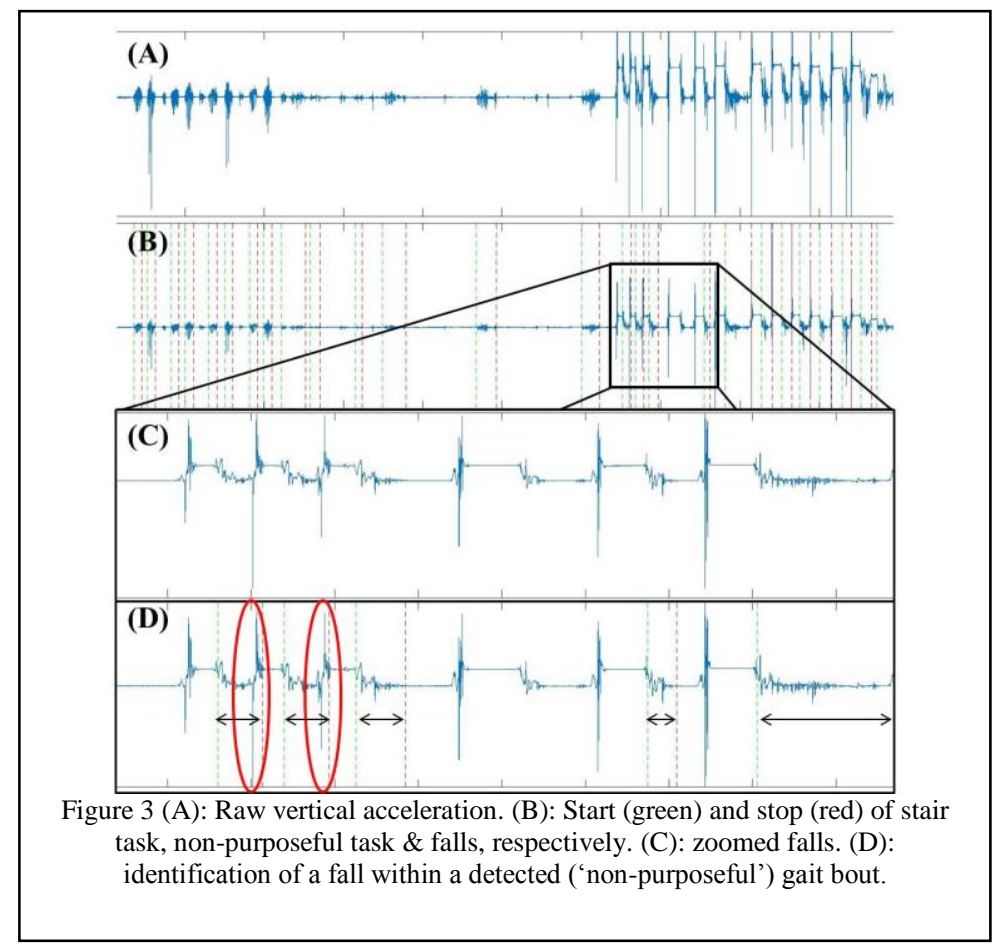

\section{B. Stage I- Falls}

The 10 healthy adults performed a total of 120 falls at the end of a period of scripted tasks, identified by the fall algorithm, Fig. 4. Sensitivity and specificity were $80.8 \%$ and $84.6 \%$, respectively. False positives (incorrect fall detection) were only observed in the fast walk ( 2 participants) and stair ascent/descent tasks (6 participants).

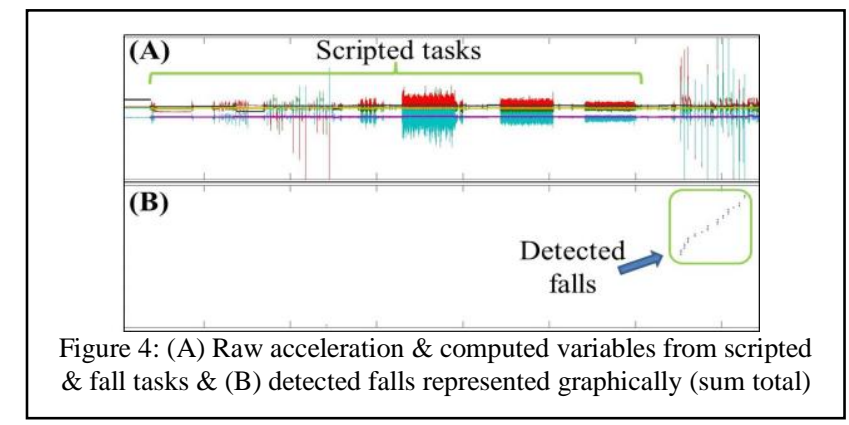

\section{B. Stage II - Participant}

The participant had low scores for balance self-efficacy ( $\mathrm{ABC}$ : 85), rated more moderate to severe for motor function (UPDRS III: 24, H\&Y-III) and had no self-reported FOG.

\section{Stage II - Gait and falls}

The falls diary reported one fall while wearing the accelerometer and described by the participant during a gait task, walking up a step. The participants 7 day accelerometer data were analysed for a fall and it was successfully detected by the algorithm at the correct day and time. In addition the pre fall event was successfully segmented using the proposed segmentation algorithm, Fig. 5(i-ii). Similar to the laboratory testing, the fall event was detected within a gait bout. However, a large number of non-verified falls were detected within the same day and remaining days (total $=38 /$ week). 


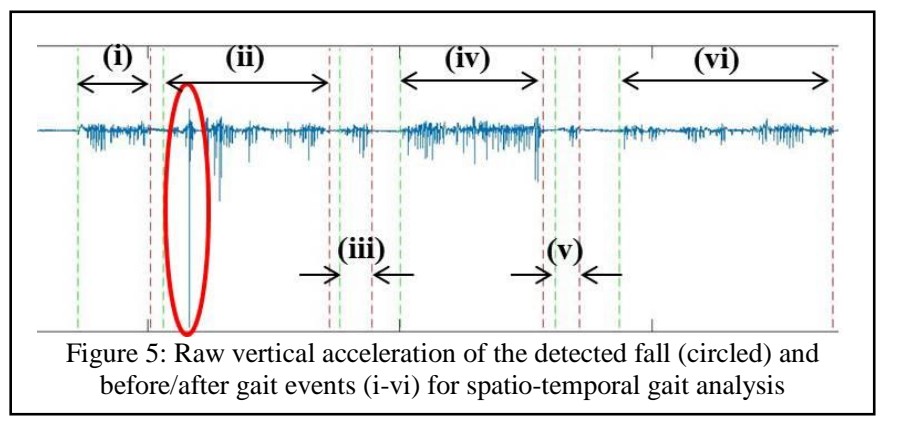

\section{Discussion}

This pilot study utilised a number of algorithms to harmonise free-living gait and fall detection within PD. We present a segmentation algorithm within a controlled setting, successfully identifying all purposeful bouts of gait as well as those deemed non-purposeful for the purposes of this study, i.e. short/abrupt steps/shuffles while waiting to complete a purposeful task. However, the exact timings of bouts and non-purposeful gait characteristics remain un-validated to video observation especially during free-living activities. This comprises the next stage of development/testing and will include longitudinal accelerometer and camera comparison.

The segmentation algorithm allowed the automated identification of an upright activity (gait) to define the gravity vector within the falls algorithm. Results for the falls algorithm were promising within a controlled setting on this independent data set, yet a number of non-fall events were observed during fast gait and stair ascent/descent, tasks not previously used to test the algorithm. Moreover, identifying and removing the fall event within a gait bout is important when considering the analysis of micro gait characteristics.

The combination of algorithms successfully identified a fall from free-living PD data compared to self-report. However, a large number of non-verified falls were detected across the 7 days. Without confirmed patient self-report of those events it is difficult to draw conclusions of validity at this early stage. Yet, the patient was moderate to severe in motor phenotype and had a long history of recurrent falls. However, examination of the raw accelerations showed similarities to the laboratory testing: non-fall events occurring during gait (identified by the segmentation algorithm), perhaps during increased gait cadence or stair ascent/descent.

Understanding free-living gait may help to reduce the number of non-fall events detected with an accelerometer. This will be achieved by understanding the context and removing the false positives, such as implementing additional context aware algorithms for stair ascent/decent [21] or understanding the micro gait characteristics (e.g. increased step time) [19] leading to or during a fall/non-fall detection.

\section{Conclusion}

Future work will implement additional algorithms (e.g. recognise stair ascent/descent) to further identify context of gait events to negate (remove) false positive generated during gait-related events as well as broaden knowledge of free-living gait. This work will also lead to a better understanding of purposeful v. non-purposeful gait events and better use of gait as a surrogate marker within PD.

\section{References}

1. Lara, J., et al., Towards measurement of the Healthy Ageing Phenotype in lifestyle-based intervention studies. Maturitas, 2013. 76(2): p. 189-99.

2. Godfrey, A. and L. Rochester, Body-worn monitors: a lot done, more to do. J Epidemiol Community Health, 2015.

3. McCamley, J., et al., An enhanced estimate of initial contact and final contact instants of time using lower trunk inertial sensor data. Gait Posture, 2012. 36(2): p. 316-8.

4. Bidargaddi, N., et al., Wavelet based approach for posture transition estimation using a waist worn accelerometer. Conf Proc IEEE Eng Med Biol Soc, 2007. 2007: p. 1884-7.

5. Bourke, A.K., J.V. O'Brien, and G.M. Lyons, Evaluation of a threshold-based tri-axial accelerometer fall detection algorithm. Gait Posture, 2007. 26(2): p. 194-9.

6. Godfrey, A., et al., iCap: Instrumented assessment of physical capability. Maturitas, 2015.

7. Weiss, A., et al., Objective assessment of fall risk in Parkinson's disease using a body-fixed sensor worn for 3 days. PLoS One, 2014. 9(5): p. e96675.

8. van Schooten, K.S., et al., Ambulatory fall-risk assessment: amount and quality of daily-life gait predict falls in older adults. J Gerontol A Biol Sci Med Sci, 2015. 70(5): p. 608-15.

9. $\quad$ Orendurff, M.S., et al., How humans walk: bout duration, steps per bout, and rest duration. J Rehabil Res Dev, 2008. 45(7): p. $1077-89$.

10. Lord, S., B. Galna, and L. Rochester, Moving forward on gait measurement: toward a more refined approach. Mov Disord, 2013. 28(11): p. 1534-43.

11. Chastin, S.F.M. and M.H. Granat, Methods for objective measure, quantification and analysis of sedentary behaviour and inactivity. Gait \& Posture, 2010. 31(1): p. 82-86.

12. Zijlstra, W. and A.L. Hof, Assessment of spatio-temporal gait parameters from trunk accelerations during human walking. Gait Posture, 2003. 18(2): p. 1-10.

13. Lord, S., et al., Independent domains of gait in older adults and associated motor and nonmotor attributes: validation of a factor analysis approach. J Gerontol A Biol Sci Med Sci, 2013. 68(7): p. 820-7.

14. Kangas, M., et al., Comparison of low-complexity fall detection algorithms for body attached accelerometers. Gait Posture, 2008. 28(2): p. 28591.

15. Bourke, A.K., et al., Evaluation of waist-mounted tri-axial accelerometer based fall-detection algorithms during scripted and continuous unscripted activities. J Biomech, 2010. 43(15): p. 3051-7. 
16. Ashburn, A., et al., The circumstances of falls among people with Parkinson's disease and the use of Falls Diaries to facilitate reporting. Disabil Rehabil, 2008. 30(16): p. 1205-12.

17. Mactier, K., et al., The relationship between real world ambulatory activity and falls in incident Parkinson's disease: influence of classification scheme. Parkinsonism Relat Disord, 2015. 21(3): p. 236-42.

18. Moe-Nilssen, R., A new method for evaluating motor control in gait under real-life environmental conditions. Part 1: The instrument. Clin Biomech (Bristol, Avon), 1998. 13(4-5): p. 320-327.

19. Del Din, S., A. Godfrey, and L. Rochester, Validation of an accelerometer to quantify a comprehensive battery of gait characteristics in healthy older adults and Parkinson's disease: toward clinical and at home use. IEEE J Biomed Health Inform, 2015.

20. Godfrey, A., et al., Instrumenting gait with an accelerometer: a system and algorithm examination. Med Eng Phys, $2015.37(4):$ p. 400-7.

21. Sekine, M., et al., Classification of waist-acceleration signals in a continuous walking record. Med Eng Phys, 2000. 22(4): p. 285-91. 